\title{
Significantly suppressing ion migration in metal halide perovskites via trace of multivalent interstitial doping
}

\section{Yepin Zhao}

University of California Los Angeles

Ilhan Yavuz

Marmara University

Minhuan Wang

Dalian University of Technology

\section{Marc Weber}

Washington State University https://orcid.org/0000-0003-4204-5875

Ju-Hong Lee

Sungkyunkwan University

Shaun Tan

University of California Los Angeles

Tianyi Huang

University of California Los Angeles

Dong Meng

Rui Wang

University of California, Los Angeles

Jingjing Xue

UCLA https://orcid.org/0000-0002-7861-8367

\section{Sung-Joon Lee}

University of California Los Angeles https://orcid.org/0000-0003-2368-4647

\section{Sang-Hoon Bae}

Washington University in St. Louis

\section{Anni Zhang}

University of California, Los Angeles

\section{Tae-Hee Han}

University of California Los Angeles

\section{Yifang Zhou}

University of California, Los Angeles

Jiming Bian

Dalian University of Technology https://orcid.org/0000-0002-4912-7606 


\section{Nam-Gyu Park}

Sungkyunkwan University https://orcid.org/0000-0003-2368-6300

Jin-Wook Lee

University of California Los Angeles

Yang Yang ( $\square$ yangy@ucla.edu )

University of California, Los Angeles https://orcid.org/0000-0001-8833-7641

\section{Article}

Keywords: ion migration, metal halide perovskites, $\mathrm{Nd} 3+$, interstitial cations

Posted Date: August 14th, 2021

DOl: https://doi.org/10.21203/rs.3.rs-803525/v1

License: (c) (i) This work is licensed under a Creative Commons Attribution 4.0 International License.

Read Full License

Additional Declarations: There is NO Competing Interest.

Version of Record: A version of this preprint was published at Nature Materials on November 17th, 2022. See the published version at https://doi.org/10.1038/s41563-022-01390-3. 


\section{materials}

\section{Significantly suppressing ion migration in metal halide perovskites via trace of multivalent interstitial doping}

Yepin Zhao ${ }^{1}$, Ilhan Yavuz ${ }^{2 *}$, Minhuan Wang ${ }^{3}$, Marc H. Weber ${ }^{4}$, Ju-Hong Lee ${ }^{5}$, Shaun Tan ${ }^{1}$, Tianyi Huang $^{1}$, Dong Meng ${ }^{1}$, Rui Wang ${ }^{1}$, Jingjing Xue ${ }^{1}$, Sung-Joon Lee ${ }^{6,7}$, Sang-Hoon Bae ${ }^{8}$, Anni Zhang ${ }^{1}$, Tae-Hee Han ${ }^{1,9}$, Yifang Zhou ${ }^{1}$, Jiming Bian ${ }^{3}$, Nam-Gyu Park ${ }^{5}$, Jin-Wook Lee ${ }^{5^{*}}$, Yang Yang ${ }^{*}$

${ }^{1}$ Department of Materials Science and Engineering and California Nano Systems Institute, University of California, Los Angeles, California 90095, United States.

${ }^{2}$ Department of Physics, Marmara University, Ziverbey, Istanbul 34722, Turkey.

${ }^{3}$ Key Laboratory of Materials Modification by Laser, Ion, and Electron Beams (Dalian University of Technology), Ministry of Education, School of Physics, Dalian, 116024 China

${ }^{4}$ Center for Materials Research, Washington State University, Pullman, WA 99164, USA

${ }^{5}$ SKKU Advanced Institute of Nanotechnology (SAINT) and Department of Nanoengineering, Sungkyunkwan University, Suwon 16419, Republic of Korea.

${ }^{6}$ Department of Chemistry and Biochemistry, University of California, Los Angeles, California 90095, United States.

${ }^{7}$ Department of Physics and Interdisciplinary Course of Physics and Chemistry, Sungkyunkwan University, Suwon 16419, Republic of Korea.

${ }^{8}$ Department of Mechanical Engineering and Materials Science, McKelvey School of Engineering, Washington University in St. Louis, St. Louis, Missouri 63130-4899, United States.

${ }^{9}$ Division of Materials Science and Engineering, Hanyang University, Seoul 04763, Republic of Korea

*Correspondence and requests for materials should be addressed to I.Y. (email: ilhan.yavuz@marmara.edu.tr) or to J.-W. L. (email: jw.lee@skku.edu) or to Y.Y. (email: yangy@ucla.edu) 
Cations with suitable sizes to occupy an interstitial site of perovskite crystals have been widely used to inhibit ion migration and promote performance and stability of perovskite optoelectronics. However, the interstitial doping accompanies inevitable lattice strain to impair long-range ordering and stability of the crystals to cause a sacrificial trade-off. Here, we unravel the evident influence of the valence states of the interstitial cations on their efficacy to suppress the ion migration. Incorporation of a trivalent neodymium cation $\left(\mathrm{Nd}^{3+}\right)$ effectively mitigates the ion migration in the perovskite lattice with significantly reduced dosage $(0.08 \%)$ compared to a widely used monovalent cation dopant $\left(\mathrm{Na}^{+}, \mathbf{0 . 4 5 \%}\right)$. Less but better, the prototypical perovskite solar cells incorporated with $\mathbf{N d}^{3+}$ exhibits significantly enhanced photovoltaic performance and operational stability.

Organometal halide perovskite solar cells have experienced eye-catching improvements in its power conversion efficiency (PCE) ${ }^{1-3}$. To implement its final step to the commercialization, functional longevity becomes the dernier continent to conquer ${ }^{4-8}$. The displacement of the ions and/or charged defects in the perovskite layer has been recognized as the major origin of device performance degradation under its working environment with electric field, heat, and excess charge carriers ${ }^{9-14}$. Therefore, the suppression of the ion migration should be the key to promote the operational stability of the perovskite solar cells.

Interstitial doping of Alkali metal cations such as lithium $\left(\mathrm{Li}^{+}\right)$, sodium $\left(\mathrm{Na}^{+}\right)$, potassium $\left(\mathrm{K}^{+}\right)$or rubidium $\left(\mathrm{Rb}^{+}\right)$have been widely used to inhibit the migration of halide ions to relieve current-voltage hysteresis and improve operational stability ${ }^{15-17}$. However, the interstitial doping possibly distorts perovskite lattice and induces strain that undermines long-range ordering and stability of the desired phase ${ }^{1,18,19}$. Perhaps for this reason, state-of-the-art record efficiency devices did not incorporate this approach. Hence, there has been a strong need to develop a new approach for preventing these side effects while maximizing the benign ion migration inhibition effect to promote both performance and the operational stability of the perovskite solar cells. Here, we demonstrate an efficient interstitial doping strategy based on trivalent neodymium cation $\left(\mathrm{Nd}^{3+}\right)$ to prominently prevent the halide migration in the perovskite lattice with a minimal dose addition. Compared with cations with similar ionic radii but the lower valences, i.e. calcium $\left(\mathrm{Ca}^{2+}\right)$ and sodium cations $\left(\mathrm{Na}^{+}\right)$, the higher valence state of $\mathrm{Nd}^{3+}$ provides better capability to obstruct the halide migration and is proven to superiorly passivate the negatively charged defects in the perovskite lattice with substantially less dopant concentration.

Effective ionic radii of $\mathrm{Na}^{+}, \mathrm{Ca}^{2+}$ and $\mathrm{Nd}^{3+}$ are 102,100 and $98.3 \mathrm{pm}$, respectively. Compared to iodide anion $\left(\mathrm{I}^{-}\right), \mathrm{Nd}^{3+}, \mathrm{Ca}^{2+}$, and $\mathrm{Na}^{+}$have cation to anion size ratios of $0.447,0.455$, 


\section{materials}

and 0.464 , respectively, and thus octahedral $\left(\mathrm{O}_{\mathrm{h}}\right)$ geometry with 6 coordination is expected as ' $\mathrm{A}$ ' site cation in $\mathrm{ABX}_{3}$ perovskite ${ }^{20}$. However, their radii are much smaller than those of $\mathrm{CH}_{3} \mathrm{NH}_{3}{ }^{+}$ $\left(\mathrm{MA}^{+}, 217 \mathrm{pm}\right)$ or $\mathrm{HC}\left(\mathrm{NH}_{2}\right)_{2}{ }^{+}\left(\mathrm{FA}^{+}, 253 \mathrm{pm}\right)^{21}$, and thus expected to occupy $\mathrm{O}_{\mathrm{h}}$ interstitial sites rather than substitution of existing $\mathrm{MA}^{+}$or $\mathrm{FA}^{+}$. We simulated the effect of the different interstitial cations on iodide ion migration energetics to the nearby iodine vacancy $\left(\mathrm{V}_{\mathrm{I}}\right)$ in perovskite crystal cells (Fig. 1A, Supplementary Note 1). Each migration route was divided into eighteen steps and the relative energy differences compared with the initial stage are recorded in Fig. 1B. Without the interstitial cation, the energy barrier for the $V_{I}$ migration was only about $0.37 \mathrm{eV}$. The presence of $\mathrm{Nd}^{3+}$ enhances the energy barrier significantly $(2.80 \mathrm{eV})$, and $\mathrm{Ca}^{2+}(0.81 \mathrm{eV})$ and $\mathrm{Na}^{+}(0.43 \mathrm{eV})$ are followed. Considering the similar sizes of the cations, the distinction of the ion migration impedance capability might be majorly related to the valence states of the cations. $\mathrm{Nd}^{3+}$ with three positive charges are more likely to restrict the motion of negative iodide ions due to stronger electrostatic attraction force. This impeded halide migration by the cation doping is favorable for the stability enhancement and hysteresis elimination of the perovskite solar cell devices.

Regardless of the beneficial ion migration mitigation effect, the interstitial cation doping can introduce tensile strain to the perovskite lattice that can potentially destabilize the lattice ${ }^{1,18,19}$. In X-ray diffraction (XRD) measurement in Fig. 1C, the progressive shift of the (100) orientation peak of $\alpha-\mathrm{FAPbI}_{3}$ to lower two theta angle with increasing cation dopant concentration supports interstitial incorporation of the cation and thus induced lattice volume expansion due to the tensile strain. According to Willamson-Hall analysis (Fig. S1-S4, Table S1-S3 and Supplementary Note 2), the lattice strain rapidly rises even with relatively low dopant concentration $(<1 \%$, by molar ratio), and then decreases with further addition probably due to relaxation by generation of dislocations, grain boundaries or secondary phases. From XRD patterns in Fig. 1D, as dopant concentration reaches $1 \%$, it was noticed that the intensity of (001) $\alpha-\mathrm{FAPbI}_{3}$ peak is reduced while that of (001) $\mathrm{PbI}_{2}$ peak intensifies. Further increase in dopant concentration to 5\% induces the appearance of (010) non-perovskite $\delta-\mathrm{FAPbI}_{3}$ peak, indicating destabilization of the $\alpha-\mathrm{FAPbI}_{3}$ perovskite phase as the concentration of the interstitial dopants increases. Scanning electron microscopic (SEM) images in Fig. S5 also showed that grain size of the film generally decreases with the addition of higher amount of cation dopant. Such generation of defects or secondary phase can rather degrade the performance and stability of the device. Thus, despite of the beneficial ion 
migration mitigation effect, minimal dopant concentration will be preferred to minimize the sacrificial trade-off.

In Fig. 2A, we assessed the photovoltaic performances of the devices with the addition of different cations. Noticeably, the optimal doping concentrations for the highest power conversion efficiencies (PCEs) were varied depending on the cations; $0.08 \%, 0.25 \%$, and $0.45 \%$ for $\mathrm{Nd}^{3+}$, $\mathrm{Ca}^{2+}$ and $\mathrm{Na}^{+}$, respectively. With $0.08 \% \mathrm{Nd}^{3+}$ doping, a distinct enhancement in the average PCE was achieved from $20.56 \pm 0.49$ to $23.31 \pm 0.29 \%$ while $0.25 \% \mathrm{Ca}^{2+}$ and $0.45 \% \mathrm{Na}^{+}$doping result in average PCEs of $22.07 \pm 0.35$ and $22.05 \pm 0.19 \%$, respectively. The open-circuit voltages ( $V_{\mathrm{OCS}}$ ), short-circuit current densities $\left(J_{S C}\right)$ and fill factors (FFs) were largely improved by the cation addition (Fig. S6-S8 and Table S4-S6). Negligible current-voltage hysteresis was observed for the device with the $\mathrm{Nd}^{3+}$ dopants whereas the $\mathrm{Ca}^{2+}$ and $\mathrm{Na}^{+}$dopants can only partially reduce the hysteresis observed from the reference device (Fig. 2B). The device with $0.08 \% \mathrm{Nd}^{3+}$ dopant showed the best PCE of $23.68 \%$ with $V_{O C}$ of $1.173 \mathrm{~V}, J_{S C}$ of $25.38 \mathrm{~mA} / \mathrm{cm}^{2}$, and FF of $79.55 \%$. The stabilized PCE is also improved from 19.56 (reference) to $23.10,22.23$ and $21.81 \%$ with $\mathrm{Nd}^{3+}$, $\mathrm{Ca}^{2+}$ and $\mathrm{Na}^{+}$doping, respectively (Fig. 2C and Table S7). External quantum efficiency (EQE) spectra of the devices were compared in Fig. 2D. An integrated $J_{\mathrm{SC}}$ of $25.12 \mathrm{mAcm}^{-2}$ from the $\mathrm{Nd}^{3+}$ doped device matched well with the value measured from the $J-V$ scan $(<5 \%$ discrepancy), whereas that of the control device $\left(24.06 \mathrm{~mA} \mathrm{~cm}^{-2}\right)$ showed relatively large discrepancy of $6.7 \%$, probably due to pronounced $J-V$ hysteresis. Fig. 2E compares the surface morphology of the perovskite films incorporated with different cation dopants. While overall grain sizes of the films are comparable, closer inspection of the images revealed that small particulates are segregated on the surface of the films with $0.25 \% \mathrm{Ca}^{2+}$ and $0.45 \% \mathrm{Na}^{+}$. We speculate relatively higher strain ( $>0.19 \%$, Table $\mathbf{S 2}$ and S3) induced by $0.25 \% \mathrm{Ca}^{2+}$ and $0.45 \% \mathrm{Na}^{+}$might cause clustering and segregation of the dopant on the crystal surface and/or grain boundary, which might limit performance enhancement by sacrificial trade-off ${ }^{22}$. On the other hand, the film with $0.08 \% \mathrm{Nd}^{3+}$ showed neat surface comparable to the reference film, and thus the side effects are probably minimized.

The interstitial cations are likely to interact with negatively charged defects. We investigate the interaction energies of the different cations with the defects including iodine-formamidinium antisites $\left(\mathrm{I}_{\mathrm{FA}}\right)$, lead vacancies $\left(\mathrm{V}_{\mathrm{Pb}}\right)$, formamidinium vacancies $\left(\mathrm{V}_{\mathrm{FA}}\right)$ and iodine-lead antisites $\left(\mathrm{I}_{\mathrm{Pb}}\right)$ based on the density functional theory (DFT) calculations (models are shown in Fig. S9). As 


\section{materials}

summarized in Fig. 3A, the interaction energies between the defects and $\mathrm{Nd}^{3+}$ was the highest and those for $\mathrm{Ca}^{2+}$ and $\mathrm{Na}^{+}$are followed. Considering the similar cation radii, the higher valence charge probably contributes to relatively stronger interaction energies of the $\mathrm{Nd}^{3+}$ with the negatively charged intrinsic defects. The stronger interaction energy of the $\mathrm{Nd}^{3+}$ might enable more effective defect mitigation with the minimal dosage for the less side effects (e.g. strain, defects, dopant segregation) and thus higher PCE of the device. The time-resolved photoluminescence (TRPL) decay curves of the films with the optimized dopant concentration are compared with that of the reference film in Fig 3B. The curves were fitted to a single exponential decay model to quantify the PL lifetime (Table S7). Overall, we observed an elongation of the PL lifetime for the films with the three cations compared with the reference perovskite film; the PL lifetimes of the $\mathrm{Nd}^{3+}$ (7.75 $\mu \mathrm{s}), \mathrm{Ca}^{2+}(5.47 \mu \mathrm{s})$, and $\mathrm{Na}^{+}(4.78 \mu \mathrm{s})$ films were significantly longer than that of the reference sample $(1.68 \mu \mathrm{s})$. Regardless of the least addition amount, the most largely elongated PL lifetime for the film with $\mathrm{Nd}^{3+}$ supports the effectiveness of the $\mathrm{Nd}^{3+}$ dopant for mitigation of the defects. The trend in PL lifetimes correlated with the enhanced steady-state PL intensity (inset of Fig. 3B). On the other hand, the cation-containing films showed no noticeable difference in their PL peak positions and absorption spectra (Fig. S10). We further investigate charge recombination kinetics in the corresponding devices by the transient photovoltage (TPV) decay measurement shown in Fig. 3C. The fitted time constants for reference, $\mathrm{Nd}^{3+}-\mathrm{Ca}^{2+}-$ and $\mathrm{Na}^{+}$-incorporated devices were $0.50,0.86,0.79$, and $0.65 \mathrm{~ms}$, respectively, which in line with the trend observed from the measured PL lifetimes.

Positron annihilation spectroscopy (PAS) was employed to compare the density of negatively or neutral charged defects in the films. The positively charged positrons are implanted from the perovskite surface, which is annihilated upon interaction with electrons from a free lattice site or after trapping at negatively charged or neutral (but not positive) vacancies and/or interstitial defects to emit two gamma photons ${ }^{23}$. The shape parameter is extracted from the doppler broadening of the gamma-ray spectrum, and it increases with the increasing density of negatively charged or neutral defects. By changing the kinetic energy of the incident positron, we were able to investigate the depth-dependent defect density of the films incorporated with different cations (Fig. 3D). The results show that the shape parameters of the films with the cations doping in the bulk (mean depth between $10 \mathrm{~nm}$ and $500 \mathrm{~nm}$, shaded region in Fig. 3D) is lower than that of the reference film, indicating a decreased density of negatively charged or neutral defects in the bulk 
region by the cation doping. The average shape parameters of different films in the bulk region are compared in Fig. 3E. The lowest shape parameter of the perovskite film with $\mathrm{Nd}^{3+}$ closely correlates with the TRPL and TPV measurements. We further cross-checked the trap density by measuring the total density of states (tDOS) for the as-fabricated devices incorporated with different cations using angular frequency-dependent capacitance measurement. As shown in Fig. 3F, the density of in-gap states for the devices with $\mathrm{Nd}^{3+}, \mathrm{Ca}^{2+}$ and $\mathrm{Na}^{+}$dopants are decreases compared with the reference device where the tendency in the measured trap density coincides with the PL and PAS measurements.

To experimentally verify the effect of the different cation dopants on the ion migration energetics, we performed a temperature-dependent conductivity measurement on lateral perovskite devices with structure of $\mathrm{Au} /$ perovskite $(100 \mu \mathrm{m}) / \mathrm{Au}$. The activation energy $\left(E_{a}\right)$ for ion migration can be extracted by linear fitting of the data based on the Nernst-Einstein equation given by $\sigma(\mathrm{T})=\frac{\sigma_{0}}{T} \exp \left(\frac{-E_{a}}{k_{b} T}\right)$, where $\sigma(\mathrm{T})$ is the conductivity as a function of temperature $\mathrm{T}, \sigma_{0}$ is a constant, $k_{b}$ is Boltzmann constant ${ }^{12}$. The $E_{\mathrm{a}}$ was extracted from the slope of the fitted lines at relatively higher temperature (Fig. 4A). The calculated $E_{a}$ for the film incorporated with $\mathrm{Nd}^{3+}$, $\mathrm{Ca}^{2+}$, and $\mathrm{Na}^{+}$were $0.812 \mathrm{eV}, 0.703 \mathrm{eV}$, and $0.626 \mathrm{eV}$, respectively, which are substantially higher than that of the reference film $(0.425 \mathrm{eV})$. The trend of the measured $E_{a}$ s also agrees well with the simulation results. The in-situ PL measurement was performed using the lateral device under 440 $\mathrm{nm}$ illumination and applied an electric field of $150 \mathrm{mV} / \mu \mathrm{m}$ to visualize the ion migration (Fig. 4B and C, Supplementary Movie 1). As time passes, an obvious PL quenching from the reference film is observed and worsens rapidly. The PL quenching is attributed to the destroyed stoichiometry and/or structure of the crystals due to pronounced migration of charged defects in the reference film ${ }^{24}$. By contrast, the bright PL signal of the film incorporated with $\mathrm{Nd}^{3+}$ remains unchanged even after applying the bias voltage for the 10 minutes, indicating the migration of the charged defects is significantly suppressed by the addition of $0.08 \% \mathrm{Nd}^{3+}$.

To investigate the operational stability of the solar cell devices, we exposed the encapsulated devices to continuous illumination under ambient atmosphere and open-circuit condition (Fig. 4D). Versus maximum power point tracking, the performance degradation is expected to be more accelerated under the open-circuit condition ${ }^{25}$. Notably, the encapsulated device incorporated with $\mathrm{Nd}^{3+}$ maintained $84.3 \%$ of its initial PCE after continuous illumination 


\section{materials}

for 2002 hours while the PCE of the reference device degraded to $0 \%$ of the initial PCE. Incorporation of $\mathrm{Ca}^{2+}$ and $\mathrm{Na}^{+}$also improved the operational stability to retain $68.0 \%$ and $48.6 \%$ of their initial PCEs, respectively. To further explore the thermal stability of the devices, we kept the unencapsulated devices in a nitrogen-filled glove box at $85^{\circ} \mathrm{C}$. After 1008 hours, the reference device completely degraded while the $\mathrm{Nd}^{3+}, \mathrm{Ca}^{2+}$, and $\mathrm{Na}^{+}$incorporated devices retained $86.4 \%$, $72.7 \%$ and $60.3 \%$ of their initial PCEs, respectively. The observed improvement in both photo and thermal stability of the devices indicates that incorporation of an interstitial cation with the stronger interaction with the charged defects is beneficial for the mitigation of defect while minimizing the side effects. Thus, regardless of the defect (impurity) tolerant nature of the perovskite, this study highlights the importance of minimizing the dosage of the interstitial dopant to maximize both photovoltaic performance and operational stability of perovskite optoelectronics.

\section{References}

1 Kim, G., Min, H., Lee, K. S., Yoon, S. M. \& Seok, S. I. Impact of strain relaxation on performance of $\alpha$-formamidinium lead iodide perovskite solar cells. Science 370, 108-112 (2020).

2 Jeong, M. et al. Stable perovskite solar cells with efficiency exceeding $24.8 \%$ and $0.3-\mathrm{V}$ voltage loss. Science 369, 1615-1620 (2020).

3 Zheng, X. et al. Managing grains and interfaces via ligand anchoring enables 22.3\%efficiency inverted perovskite solar cells. Nature Energy 5, 131-140 (2020).

4 Lin, Y.-H. et al. A piperidinium salt stabilizes efficient metal-halide perovskite solar cells. Science 369, 96-102 (2020).

5 Liu, Z. et al. A holistic approach to interface stabilization for efficient perovskite solar modules with over 2,000-hour operational stability. Nature Energy 5, 596-604 (2020).

6 Tan, H. et al. Efficient and stable solution-processed planar perovskite solar cells via contact passivation. Science 355, 722-726 (2017).

7 Jung, E. H. et al. Efficient, stable and scalable perovskite solar cells using poly (3hexylthiophene). Nature 567, 511-515 (2019).

8 Wang, L. et al. A Eu3+-Eu2+ ion redox shuttle imparts operational durability to $\mathrm{Pb}-\mathrm{I}$ perovskite solar cells. Science 363, 265-270 (2019).

9 Yuan, Y. \& Huang, J. Ion migration in organometal trihalide perovskite and its impact on photovoltaic efficiency and stability. Accounts of chemical research 49, 286-293 (2016).

10 Calado, P. et al. Evidence for ion migration in hybrid perovskite solar cells with minimal hysteresis. Nature communications 7, 1-10 (2016).

11 Tan, S. et al. Steric impediment of ion migration contributes to improved operational stability of perovskite solar cells. Advanced Materials 32, 1906995 (2020).

12 Lee, J.-W., Kim, S.-G., Yang, J.-M., Yang, Y. \& Park, N.-G. Verification and mitigation of ion migration in perovskite solar cells. APL materials 7, 041111 (2019).

13 Zhang, H. et al. Phase segregation due to ion migration in all-inorganic mixed-halide perovskite nanocrystals. Nature communications 10, 1-8 (2019). 


\section{materials}

14 Boyd, C. C., Cheacharoen, R., Leijtens, T. \& McGehee, M. D. Understanding degradation mechanisms and improving stability of perovskite photovoltaics. Chemical reviews 119, 3418-3451 (2018).

15 Abdi-Jalebi, M. et al. Maximizing and stabilizing luminescence from halide perovskites with potassium passivation. Nature 555, 497-501 (2018).

16 Son, D.-Y. et al. Universal approach toward hysteresis-free perovskite solar cell via defect engineering. Journal of the American Chemical Society 140, 1358-1364 (2018).

17 Cao, J., Tao, S. X., Bobbert, P. A., Wong, C. P. \& Zhao, N. Interstitial occupancy by extrinsic alkali cations in perovskites and its impact on ion migration. Advanced Materials 30, 1707350 (2018).

18 Jones, T. W. et al. Lattice strain causes non-radiative losses in halide perovskites. Energy \& Environmental Science 12, 596-606 (2019).

19 Zhao, J. et al. Strained hybrid perovskite thin films and their impact on the intrinsic stability of perovskite solar cells. Science advances 3, eaao5616 (2017).

20 Callister, W. D. Fundamentals of materials science and engineering. Vol. 471660817 (Wiley London, 2000).

21 Kim, J. Y., Lee, J.-W., Jung, H. S., Shin, H. \& Park, N.-G. High-efficiency perovskite solar cells. Chemical Reviews 120, 7867-7918 (2020).

22 Yan, M., Cannon, R. \& Bowen, H. Space charge, elastic field, and dipole contributions to equilibrium solute segregation at interfaces. Journal of Applied Physics 54, 764-778 (1983).

23 Tan, S. et al. Shallow Iodine Defects Accelerate the Degradation of $\alpha$-Phase Formamidinium Perovskite. Joule 4, 2426-2442 (2020).

24 Kim, S.-G. et al. Potassium ions as a kinetic controller in ionic double layers for hysteresisfree perovskite solar cells. Journal of Materials Chemistry A 7, 18807-18815 (2019).

25 Domanski, K., Alharbi, E. A., Hagfeldt, A., Grätzel, M. \& Tress, W. Systematic investigation of the impact of operation conditions on the degradation behaviour of perovskite solar cells. Nature Energy 3, 61-67 (2018).

\section{Acknowledgments}

Funding: This material is based upon work supported by the U.S. Department of Energy's Office of Energy Efficiency and Renewable Energy (EERE) under the Solar Energy Technologies Office Award Number DE-EE0008751. J.-W.L. acknowledges financial support from the National Research Foundation of Korea (NRF) grant funded by the Korea government (MSIT) under contract number 2020R1F1A1067223. Computing resources used in this work were provided by the National Center for High Performance Computing (UHEM) of Turkey (Grant No. 1008342020). I. Y. acknowledges support by the Scientific and Technological Research Council of Turkey (TÜBITAK) (Grant no: 119F380).

Author contributions: Y.Z. and J.-W.L. conceived the idea, designed and conducted the experiments, and prepared the manuscript under the supervision of Y.Y.. I.Y. performed the DFT calculations. M.W. collected SEM and XPS data under the supervision of J.B.. M.H.W. performed 


\section{materials}

PAS test and analyzed the data. S.T. helped with XRD tests. T.H. performed TPC, TPV tests. S.J.L. measured DC temperature-dependent conductivity of the samples. A.Z. helped with film optimization. R.W., J.X., T.-H. H., S.-H. B. and N.-G. P. provided helpful discussion during the project. All the authors discussed the results and commented on the manuscript.

Competing interests: Authors declare no competing interests.

Data and materials availability: All data are available in the main text or the supplementary materials.

\section{Supplementary Materials:}

Materials and Methods

Supplementary Text S1-S6

Figs. S1-S10

Tables S1 


\section{materials}

A
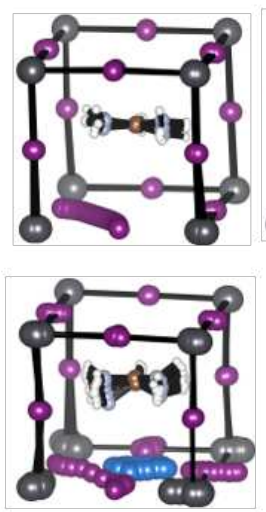

B

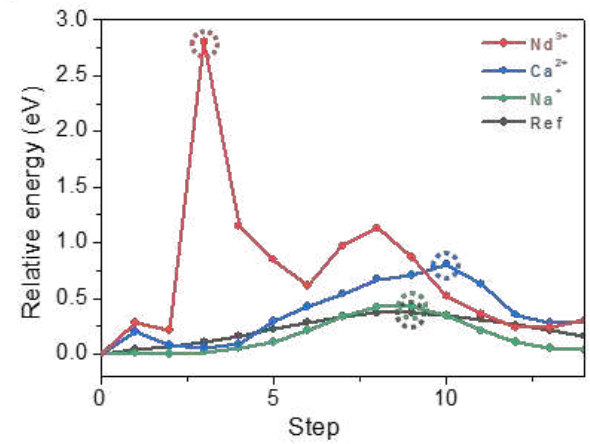

C

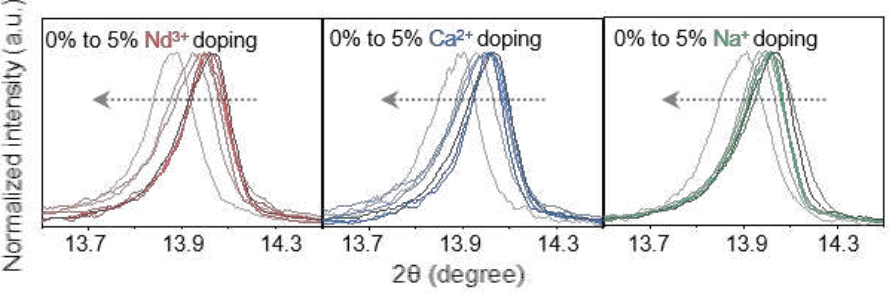

D

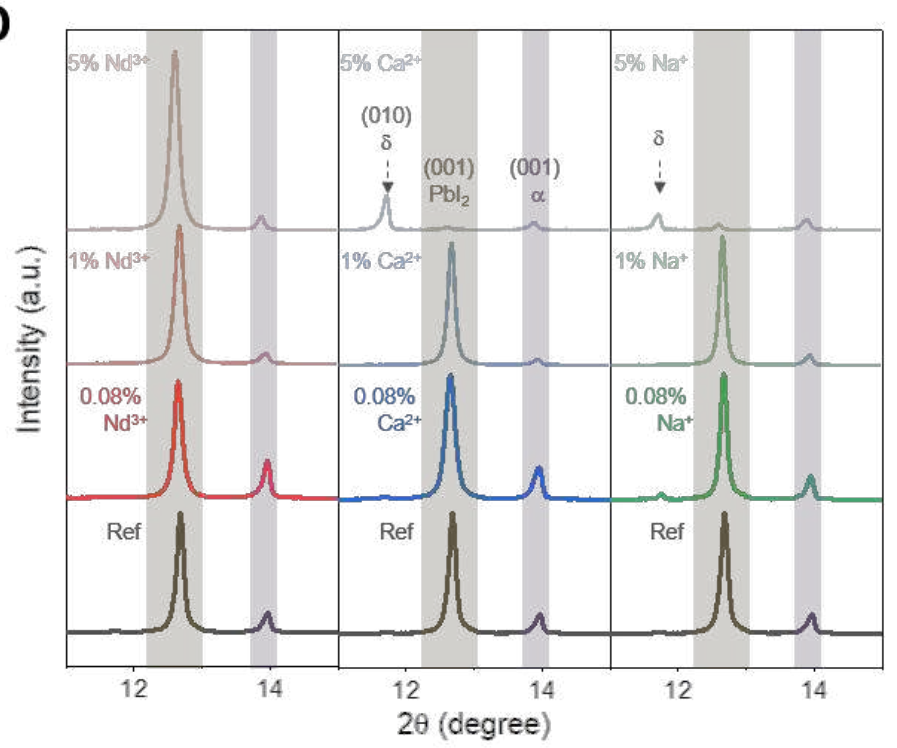

Fig. 1 | (A) Theoretical models of iodide ion migration pathway in perovskite lattices without or with $\mathrm{Nd}^{3+}, \mathrm{Ca}^{2+}$, or $\mathrm{Na}^{+}$at the interstitial site. (B) Relative energy landscapes of the system during the iodide ion migration. (C) $\alpha-\mathrm{FAPbI}_{3}$ (100) peaks from the XRD spectra of perovskite films as a function of $\mathrm{Nd}^{3+}, \mathrm{Ca}^{2+}$, and $\mathrm{Na}^{+}$doping concentration from $0 \%$ to $5 \%$. The arrows indicate a shift of the peak toward lower two theta angle with increasing dopant concentration (D) Comparison of the $\alpha-\mathrm{FAPbI}_{3}, \delta-\mathrm{FAPbI}_{3}$, and $\mathrm{PbI}_{2}$ phase variations from the XRD spectra of films with different $\mathrm{Nd}^{3+}, \mathrm{Ca}^{2+}$, and $\mathrm{Na}^{+}$doping concentration. 


\section{materials}

A
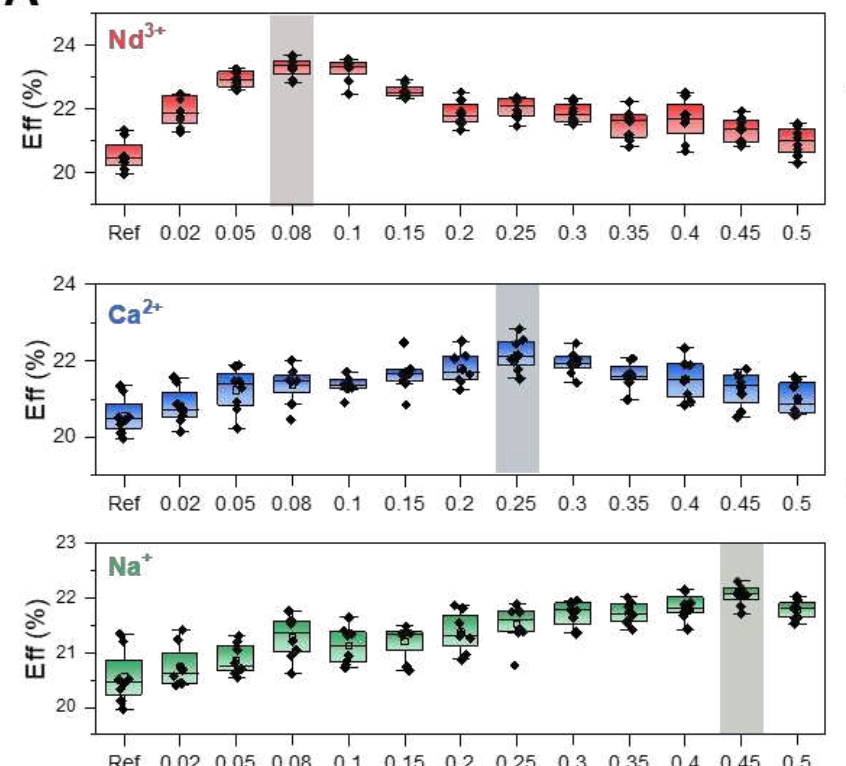

E
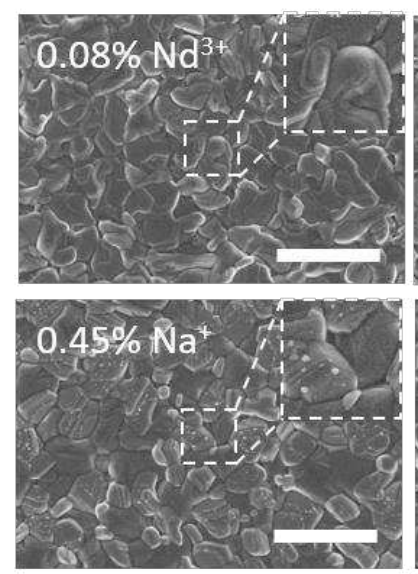

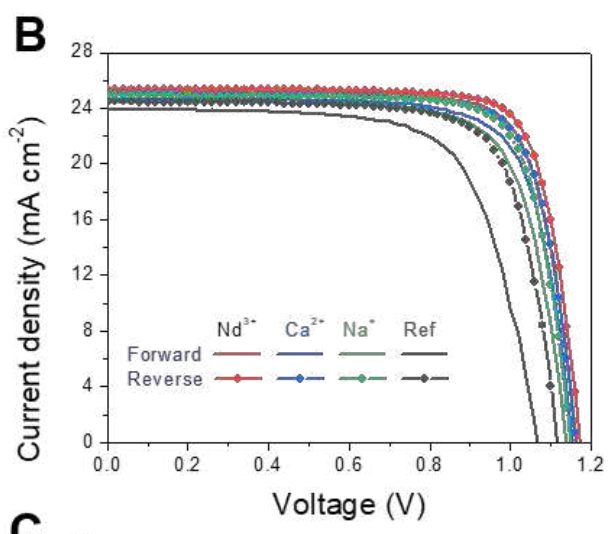

C
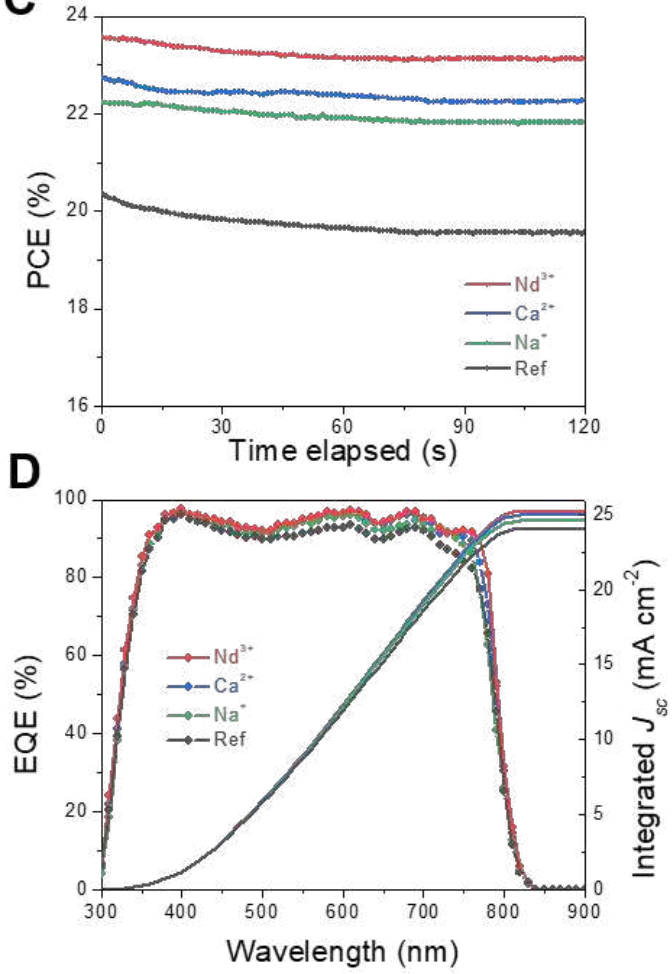

Fig. 2 (A) Statistical PCEs of devices with different concentrations of $\mathrm{Nd}^{3+}, \mathrm{Ca}^{2+}$, or $\mathrm{Na}^{+}$ incorporation. The optimal concentrations to achieve the highest PCE for each dopant are highlighted. (B) $J-V$ curves, (C) steady-state PCE measurement results and (D) EQE spectra of the best-performing devices for each condition. (E) SEM surface morphology of the perovskite films without or with the optimal cation doping concentration. 

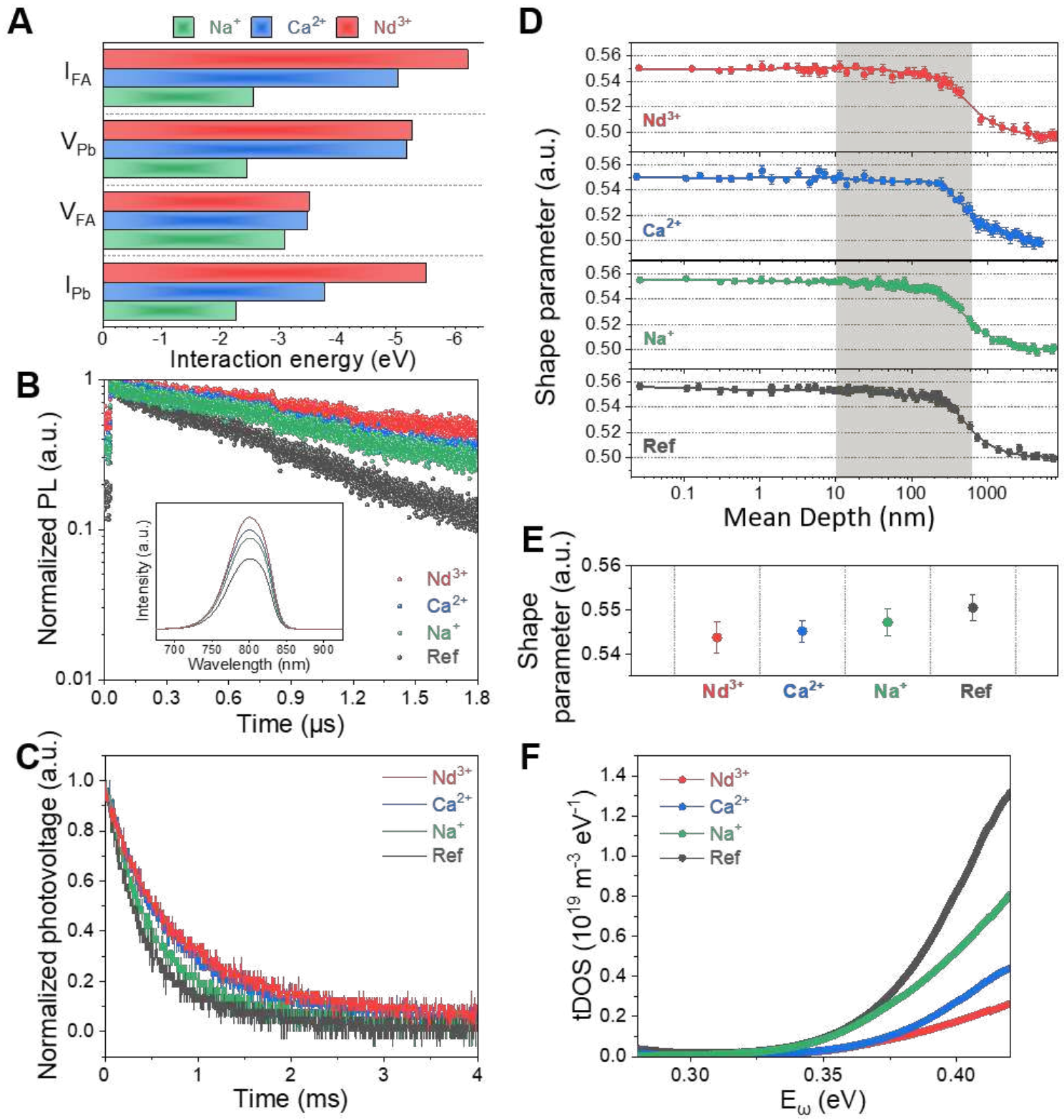

Fig. 3 (A) Interaction energies of $\mathrm{Nd}^{3+}, \mathrm{Ca}^{2+}$, or $\mathrm{Na}^{+}$cations incorporated in the perovskite lattices with the negatively charged defects. (B) Time-resolved PL spectra of perovskite films without or with the optimal cation doping concentrations (insert: corresponding steady-state spectra of perovskite films). (C) Transient photovoltage (TPV) curves of perovskite films without or with the optimal cation doping concentrations. (D) PAS depth-profiling of perovskite films without or with the optimal cation doping concentrations. Solid lines are fitted plots. Shaded areas indicate the top surface region of the films. (E) Average shape parameters in the bulk region extracted from the PAS measurement $(\mathbf{F})$ tDOS in perovskite solar cells with or without cation incorporation. 


\section{materials}

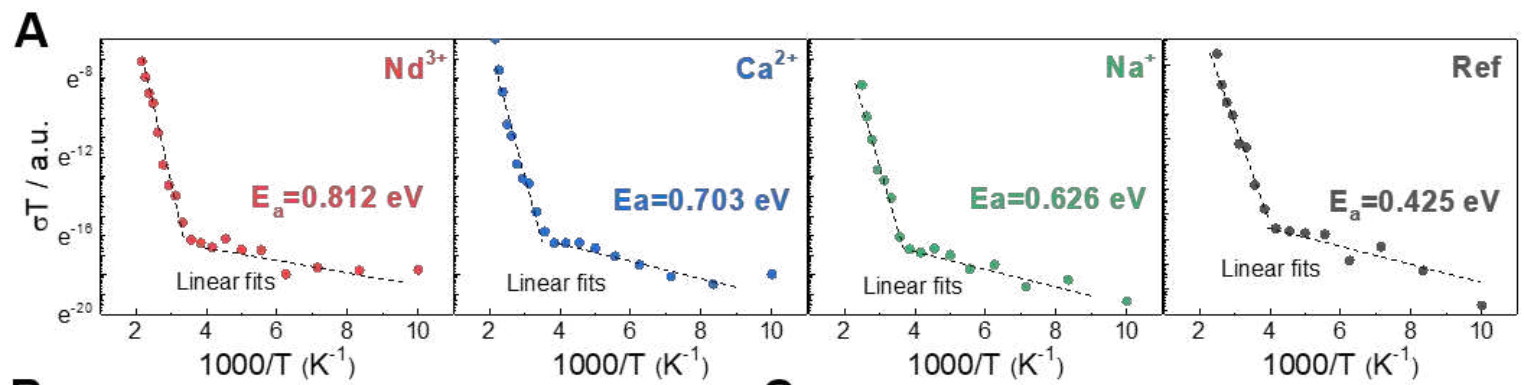

B

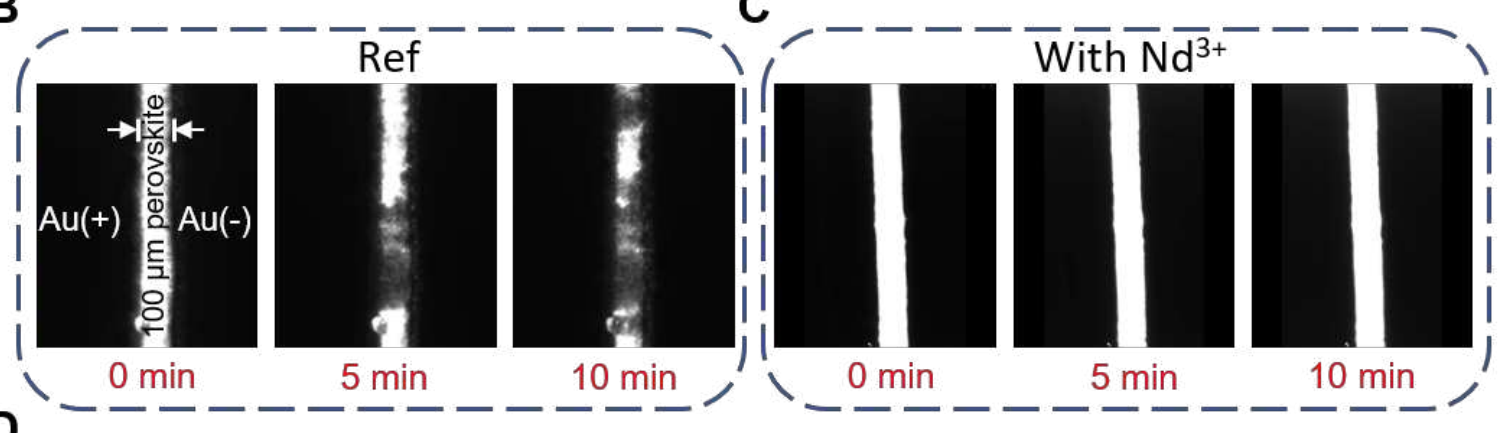

D

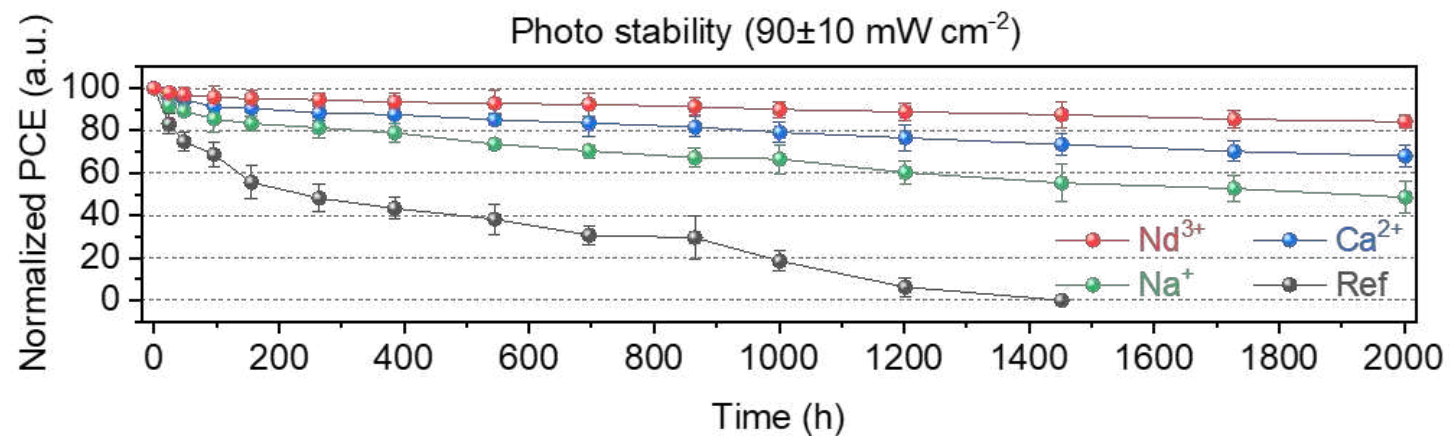

E

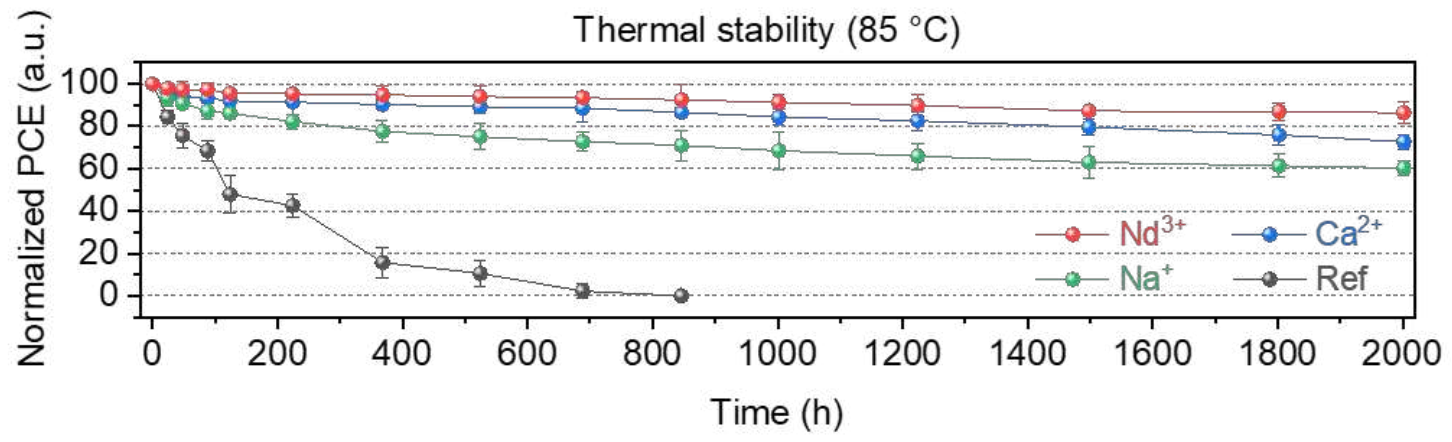

Fig. 4 | (A) Temperature-dependent conductivity of the lateral devices with $\mathrm{Nd}^{3+}, \mathrm{Ca}^{2+}$, or $\mathrm{Na}^{+}$ incorporations or without cation incorporation. Filled circles are measured data while dashed lines are fitted curves. (B, C) In situ PL images before and after applying an electric field of $150 \mathrm{mV} / \mu \mathrm{m}$ under $440 \mathrm{~nm}$ illumination for 5 and 10 min for the lateral device based on (B) the reference film and $(\mathbf{C})$ the film incorporated with $0.08 \% \mathrm{Nd}^{3+}$. (D) Photo stability of perovskite solar cells without 


\section{materials}

or with the optimal cation doping concentrations. (E) Thermal stability of perovskite solar cells without or with the optimal cation doping concentrations. 


\section{Supplementary Files}

This is a list of supplementary files associated with this preprint. Click to download.

- SupplementaryMovie1.mp4

- SupplementaryMaterial.pdf 